\title{
Position Change During Sleep Period in Patients with Multiple System Atrophy
}

\author{
Hyunwoo Nam, MD, Yoon-Ho Hong, MD, Hyung-Min Kwon, MD, Jinwhan Cho, MD \\ Department of Neurology, Boramae Hospital, Seoul National University College of Medicine
}

Background and Purpose: We wanted to investigate whether patients with multiple system atrophy (MSA) change their positions less frequently compared to the controls during sleep and during the sleep period time.

Methods: Fifteen MSA patients and fifteen controls were compared. They were matched in sex, age, apnea-hypopnea index (AHI), and PLMI (periodic limb movements index during sleep). Technician-attended standard polysomnography was performed. Position changes were divided into 3 categories depending on the interval between the arousal and the start of the position change. Category 1 was the position changes that initiated within 3 seconds after arousal. Category 2 was the position changes that occurred between 3 and 10 seconds of arousal and category 3 was the position change after 10 seconds of arousal.

Results: As a whole, position changes are less frequent in the MSA group. Category 1 position change is decreased in stages 1 and REM in MSA group compared to the control group. The frequency of category 2 position change is also dramatically lower in the MSA group as a whole and especially with prior stages 1 and 2. Category 3 position change shows marked difference between the two groups when they are compared as position change per hour of WASO.

Conclusions: Our study shows less frequent position changes during sleep in MSA patients. Longer period of wakefulness during night seems to be a compensatory mechanism for the position change.

Key Words : Multiple system atrophy, Position change, Sleep, Polysomnography

\section{Introduction}

Parkinsonian patients are known to have many sleep complains. $^{1-8}$ Tandberg et al. ${ }^{9}$ in his community-based study of 245 Parkinson's disease (PD) patients, reported two thirds were complaining of sleep problems compared with $33 \%$ of the healthy controls. Sleep fragmentation and early awakening were the main complaints. Lees et al. ${ }^{10}$ surveyed severely affected PD patients and nearly all the patients had sleep complaints. Multiple system atrophy (MSA), one of the synucleinopathy like PD, is also known to have the similar but more severe sleep complaints. ${ }^{11}$ Both in PD

\footnotetext{
* Address of correspondence

Jinwhan Cho, M.D.

Department of Neurology, Boramae Hospital

Shindaebang-2-Dong 395, Dongjak-Ku, Seoul, 156-707, Korea

Tel: +82-2-840-2507 Fax: +82-2-831-2826

E-mail: praisecho@hanmail.net
}

and MSA, various phenomena can cause patients' nocturnal sleep problems. Difficulty turning in bed has been regarded as one of the causative factors of poor sleep as staying in one position for a long time causes discomfort and pain. ${ }^{12}$ However, there have been few systematic studies dealing with this matter. Laihinen et al. ${ }^{13}$ studied body movements during sleep in 9 PD patients using staticcharge sensitive bed. They found that PD patients moved less frequently than the normal controls. Weller et al. ${ }^{14}$ used unique rotational sensor and drew the same conclusion. Contrary to these two studies, van Hilten et al., ${ }^{15}$ by their questionnaire study, reported that only 4 of 90 PD patients complained problems to turn over in bed and concluded that it was not a significant figure. In the aspect of methodology, the results are all inconclusive. In addition, studies with MSA patients are completely lacking. Though theoretically reasonable and frequently cited in 
the textbooks, decreased position change during sleep in parkinsonian patients need to be confirmed through polysomnography (PSG) as reports till now are mainly from results of unobserved tests. Here, we want to compare MSA patients and controls and hope to reveal the difference in the change of position during sleep through the standard PSG.

\section{Subjects and Methods}

MSA patients and controls were recruited from June, 2004 to January, 2007. MSA was diagnosed by a neurologist who was a movement disorder specialist. He used "probable MSA" in the diagnostic criteria. ${ }^{16}$ Controls were selected from our database of patients who underwent PSG also from June, 2004 to January, 2007. They were sex-, age-, apnea-hypopnea index (AHI)-, and PLMI (periodic limb movements index during sleep)-matched with the MSA patients. All the MSA patients and controls were free from neurological, medical, and sleep disorders other than the obstructive sleep apnea (OSA) and periodic limb movements during sleep (PLMS). Ten out of 15 MSA patients additionally showed muscle hypertonia with or without visible jerky movements during REM sleep indicating REM sleep behavior disorder. Each group had 7 males and 8 females and the mean age was 59.9 years in the MSA group and 55.3 years in the control group. (Table 1) Mean AHI was 5.9 and 6.6 and mean PLMI was 32.2 and 29.3 each.

On the night of PSG, patients were asked a list of questions including the habitual sleep schedule and complaints related to sleep such as of insomnia, excessive daytime sleepiness. In addition, information about the drug use, past medical history, and family history was collected.

We used Beehive-7 and Comet systems to record the PSG for one night in each patient (Grass-Telefactor, USA). EEG was recorded in 4 channels (C3-A2, C4-A1, O1-A2,
O2-A1), electrooculogram in 2 channels, and submental EMG in 2 channels. Data from a chest belt and an abdominal belt, a nasal and an oral thermistors, a pulse oximeter, and a neck mike were also recorded. To monitor the PLMS, surface electrodes were attached to the bilateral tibialis anterior muscles. Other monitoring devices included EKG electrodes, a body position sensor, and an infrared video camera.

Gold-plated ball bearing rotary sensor detected subject's body position. This sensor gave out $0 \mathrm{~V}$ in up, $0.41 \mathrm{~V}$ in supine, $0.78 \mathrm{~V}$ in right lateral, $0.62 \mathrm{~V}$ in prone, and 0.19 $\mathrm{V}$ in left lateral position. The body position sensor changed voltages at 45 degrees going from one position to the other with a tolerance of \pm 15 degrees. If a patient was in supine position and turned to the left, the sensor changed voltage as he was approximately halfway to the left.

Sleep recordings were staged by a neurologist who was specialized in the sleep disorder according to the Rechtschaffen and Kales criteria. ${ }^{17}$ Apnea was defined as $90 \%$ or more reduction of airflow for 10 seconds or more with either arousal in EEG or decrease of oxygen saturation of $4 \%$ or more. Hypopnea was defined as $30 \%$ or more reduction of airflow for 10 seconds with $4 \%$ or more decrease of oxygen saturation. AHI was the sum of the apnea and hypopnea per hour during sleep. PLMS were scored when the tibialis anterior EMG showed 4 or more repetitive waves with 0.5 -5-second duration and 5-90-second interval. Arousal was scored when there was an abrupt shift in EEG frequency to theta or alpha range or to frequencies greater than $16 \mathrm{~Hz}$ but not spindle.

In order to analyze the position changes, we divided the position change into 3 categories. Category 1 was the position changes that initiated within 3 seconds after arousal. We presumed this as occurring during sleep. Category 2 was the position changes that occurred between 3 and 10 seconds of arousal. We assumed the purpose of arousal in this category was to change position and thus analyzed the sleep stages before the subject woke to move. 
Category 3 was the position change after 10 seconds of arousal. We thought that the arousal and the position change were not related in this category and the analysis of prior sleep stages was meaningless.

To compare the sleep patterns between the MSA and the control groups, we used the $\chi^{2}$ test for the dichotomized data and the student's $t$-test for the continuous data. Probability values were 2-tailed, and values of $p<$ 0.05 were considered significant. All statistical analyses were performed using SPSS $12 \mathrm{~K}$ for Windows (SPSS Inc).

\section{Results}

Table 1 is describing demographic features of the two groups. Naturally from our selection criteria, there is no difference in age, sex, AHI, and PLMI between the groups. Body mass index and arousal index don't show any difference. As to the PSG parameters, there is no difference in the percentage of each sleep stage. Differences are found in total sleep time (TST), wake after sleep onset (WASO), and sleep efficiency (SE). MSA group shows less TST, more WASO, and lower SE compared to the control group.

MSA patients tend to sleep in supine position (Table 2). Contrary to the relatively even distribution of sleeping in left and right lateral and supine positions in the control group, MSA patients sleep predominantly in the supine position and the proportion of left lateral position was extremely low. As the time spent in each stage was different, we compared both groups by position change per hour of a stage. During sleep, position change is significantly less frequent in the MSA group. There are significantly less category 1 position changes in stages 1 and REM in MSA group compared to the control group. The frequency of the category 2 position changes is also dramatically decreased in the MSA group as a whole and especially in sleep stages 1 and 2. Position change per

Table 1. Demographic features and basic PSG parameters

\begin{tabular}{|c|c|c|c|}
\hline & Control $(n=15)$ & MSA $(n=15)$ & $\mathrm{P}$ value \\
\hline Age, years & $55.3 \pm 10.9$ & $59.9 \pm 7.8$ & 0.195 \\
\hline Sex, male & $7(47 \%)$ & $7(47 \%)$ & 1.000 \\
\hline BMI & $24.27 \pm 3.6$ & $23.0 \pm 2.4$ & 0.268 \\
\hline Hoehn \& Yahr stage & 0 & $3.5(3-4)$ & \\
\hline L-dopa equivalent dose (mg) & 0 & $378(0-750)$ & \\
\hline TST (min) & $351.0 \pm 68.8$ & $248.4 \pm 86.8$ & 0.001 \\
\hline Stage $1(\%$ TST $)$ & $16.9 \pm 9.0$ & $18.3 \pm 10.2$ & 0.696 \\
\hline Stage $2(\%$ TST $)$ & $49.7 \pm 12.3$ & $45.1 \pm 9.6$ & 0.265 \\
\hline Stage $3(\%$ TST $)$ & $8.0 \pm 3.9$ & $11.7 \pm 6.3$ & 0.066 \\
\hline Stage 4 (\% TST) & $7.0 \pm 7.7$ & $9.6 \pm 10.9$ & 0.460 \\
\hline Stage REM $(\%$ TST $)$ & $18.4 \pm 7.5$ & $15.3 \pm 9.3$ & 0.330 \\
\hline Sleep period time $(\min )$ & $414.3 \pm 53.4$ & $399.9 \pm 50.2$ & 0.455 \\
\hline Wake after sleep onset (min) & $63.3 \pm 37.7$ & $151.5 \pm 54.6$ & $<0.001$ \\
\hline Sleep efficiency $(\%)$ & $81.6 \pm 10.7$ & $57.2 \pm 15.9$ & $<0.001$ \\
\hline AI (No. per hour) & $16.4 \pm 8.5$ & $16.3 \pm 11.3$ & 0.970 \\
\hline AHI (No. per hour) & $6.62 \pm 7.96$ & $5.90 \pm 6.99$ & 0.794 \\
\hline PLMI (No. per hour) & $29.34 \pm 45.86$ & $32.24 \pm 56.46$ & 0.878 \\
\hline
\end{tabular}

Values are means $\pm \mathrm{SD}$, mean (range) or No. of participants (percentage).

$P$ values were obtained using the $\chi^{2}$ test for categorical data, and the student $t$-test for continuous data.

BMI, body mass index; TST, total sleep time; AI, arousal index; AHI, apnea-hypopnea index; PLMI, periodic limb movement index 
Table 2. Sleep position and position changes

\begin{tabular}{|c|c|c|c|}
\hline & Control $(n=15)$ & MSA $(n=15)$ & $\mathrm{P}$ value \\
\hline \multicolumn{4}{|l|}{ Sleep Position } \\
\hline Left & $23.0 \pm 20.5$ & $3.7 \pm 11.9$ & 0.004 \\
\hline Right & $37.9 \pm 30.7$ & $23.6 \pm 36.1$ & 0.250 \\
\hline Supine & $37.5 \pm 28.5$ & $72.7 \pm 38.3$ & 0.008 \\
\hline Prone & $0.1 \pm 0.5$ & $0.0 \pm 0.0$ & 0.334 \\
\hline \multicolumn{4}{|l|}{ Category 1} \\
\hline PC/stage 1 & $0.91 \pm 1.10$ & $0.12 \pm 0.45$ & 0.019 \\
\hline PC/stage 2 & $0.38 \pm 0.54$ & $0.12 \pm 0.33$ & 0.116 \\
\hline PC/slow wave sleep & $0.37 \pm 0.94$ & $0.00 \pm 0.00$ & 0.146 \\
\hline PC/stage REM & $0.92 \pm 1.15$ & $0.06 \pm 0.25$ & 0.013 \\
\hline $\mathrm{PC} / \mathrm{TST}$ & $0.53 \pm 0.48$ & $0.07 \pm 0.16$ & 0.003 \\
\hline \multicolumn{4}{|l|}{ Category 2} \\
\hline $\mathrm{PC} / \mathrm{SPT}(<10 \mathrm{~s})$ & $0.69 \pm 0.53$ & $0.06 \pm 0.13$ & $<0.001$ \\
\hline PC/WASO $(<10 \mathrm{~s})$ & $5.64 \pm 6.07$ & $0.17 \pm 0.38$ & 0.004 \\
\hline PC/Prior stage 1 & $1.22 \pm 1.09$ & $0.23 \pm 0.52$ & 0.005 \\
\hline $\mathrm{PC} /$ Prior stage 2 & $0.71 \pm 0.81$ & $0.04 \pm 0.15$ & 0.007 \\
\hline PC/Prior slow wave sleep & $0.42 \pm 0.69$ & $0.11 \pm 0.42$ & 0.147 \\
\hline PC/Prior stage REM & $1.33 \pm 2.05$ & $0.18 \pm 0.69$ & 0.055 \\
\hline \multicolumn{4}{|l|}{ Category 3} \\
\hline $\mathrm{PC} / \mathrm{SPT}(>10 \mathrm{~s})$ & $1.10 \pm 1.29$ & $0.85 \pm 0.90$ & 0.550 \\
\hline PC/WASO $(>10 \mathrm{~s})$ & $6.38 \pm 5.07$ & $1.99 \pm 2.00$ & 0.006 \\
\hline \multicolumn{4}{|l|}{ Total } \\
\hline Total PC/SPT & $1.79 \pm 1.57$ & $0.91 \pm 0.98$ & 0.078 \\
\hline
\end{tabular}

Values are means $\pm \mathrm{SD}$ or No. of participants (percentage).

PC, position change; TST, total sleep time; SPT, sleep period time; WASO, wake after sleep onset

hour of WASO shows significant difference, too. Category 3 position change shows marked difference between the two groups when they are compared as position change per hour of WASO.

\section{Discussion}

Decreased position change has been considered as one of the main cause of sleep disturbances in parkinsonian patients. Long period in a single position is proposed to cause pain in the contacted area, thus eliciting frequent arousal and fragmented sleep. As lack of mobility is one of the characteristics in patients of parkinsonism, researchers have taken the above theory granted. However, there has been little evidence that support the previous belief. Laihinen et al. ${ }^{13}$ used static-charge sensitive bed for the purpose of detecting movements in parkinsonian patients. The turningover events were detected by the amplitude and waveform of respiratory movements and the changes in ballistocardiogram. Weller et al. ${ }^{14}$ devised a novel rotation sensor and applied it to the old aged and to the patients with parkinsonism. van Hilten ${ }^{15}$ used a questionnaire to obtain information about the problems in turning in bed in patients with Parkinson's disease. These studies have some limitations in common. First, the studies didn't use current standard PSG and the technician didn't attend throughout the data collection. Second, detailed analysis was missing in the aspect of sleep medicine as the purposes were to test the new methods and not to analyze 
patients' sleep. We performed the standard PSG procedures with an attending technician. He observed patients' states during the recording and any errors and ambiguities could be immediately corrected. Thus our data is more reliable and more accurate.

As to the sleeping position, MSA patients tend to sleep in a supine position. This seems to be a result of decreased motility during sleep. Patients began the PSG in a supine position and the center of gravity is lowest in a supine position. Consequently, it is easy to change positions from lateral decubitus to supine but it is difficult to do in a reverse way.

We divided the position change into three categories depending on the duration of arousal before the subjects moved. Though some researchers think that shorter than 3 seconds is sufficient for the arousal, 3 seconds of EEG change is the usual criteria to score the arousal. Thus, we applied this criterion to differentiate position changes while asleep and during wakefulness. We additionally set a criterion of 10 seconds of arousal to separate categories 2 and 3. This is somewhat arbitrary. However, we thought that it was necessary because arousal for the position change and position change after arousal were different. We regarded arousal followed by immediate position change within 10 seconds as arousal for the position change. If the subjects changed their positions long after arousal, i.e. after more than 10 seconds, we considered it as moved during wakefulness and not as related to the sleep.

Our results confirm that MSA patients change their positions less frequently compared to non-MSA controls. This is evident in all categories. In category 1 , this difference is significant during stages 1 and REM, and as a whole. In category 2 , it is marked with prior stages 1 and 2 and also as a whole. Because subjects move very rarely during deep sleeps, we should have needed more subjects to detect the small difference. However, the tendency of more position changes in normal controls than MSA patients in deeper sleeps is the same as that in lighter sleeps.
We think that it is appropriate to compare the category 3 as position change per hour of WASO because, when the subjects are aroused longer than 10 seconds, the epoch of position change tends to be scored as wake. The control group's position change is significantly frequent compare to the MSA group. As position changes are more frequent in the category 3 in both groups and the sleep efficiency is lower in the MSA group, it is understandable that there is no statistical difference of position change per hour of sleep period time. MSA patients wake more time during the sleep period than the controls and the position changes occur more frequently during wake period than during sleep. This leads to a marginally significant difference in the number of position changes between the two groups as a whole. This also lets us suspect that low SE is a compensatory mechanism for the position change.

In conclusion, our study shows less frequent position changes during sleep in MSA patients. However, longer period of wakefulness makes them compensate their position changes during the whole night.

\section{REFERENCES}

1. Kumar S, Bhatia M, Behari M. Sleep disorders in Parkinson's disease. Mov Dis 2002;17(4):775-781.

2. Brodsky MA, Godbold J, Roth T, Olanow CW. Sleepiness in Parkinson's disease: a controlled study. Mov Dis 2003;18(6): 668-672.

3. Tandberg E, Larsen JP, Karlsen K. Excessive daytime sleepiness and sleep benefit in Parkinson's disease: a community-based study. Mov Dis 1999;14(6):922-927.

4. Pappert EJ, Goetz CG, Niederman FG, Raman R, Leurgans S. Hallucinations, sleep fragmentation, and altered dream phenomena in Parkinson's disease. Mov Dis 1999;14(1):117-121.

5. Ondo WG, Vuong KD, Khan H, Atassi F, Kwak C, Jankoic J. Daytime sleepiness and other sleep disorders in Parkinson's disease. Neurology 2001;57:1392-1396.

6. Wetter TC, Collado-Seidel V, Pollmacher T, Yassouridis A, Trenkwalder C. Sleep and periodic leg movement patterns in drugfree patients with Parkinson's disease and multiple system atrophy. Sleep 2000;23(3):1-7.

7. Vetrugno R, Provini F, Cortelli P, Plazzi G, Lotti EM, Pierangeli $\mathrm{G}$, et al. Sleep disorders in multiple system atrophy: a correlative 
video-polysomnographic study. Sleep Med 2004;5:21-30.

8. Plazzi G, Corsini R, Provini F, Pierangeli G, Martinelli P, Montagna $\mathrm{P}$, et al. REM sleep behavior disorders in multiple system atrophy. Neurology 1997;48(4):1094-1097.

9. Tandberg E, Larsen JP, Karlsen KH. A community-based study of sleep disorders in patients with Parkinson's disease. Mov Disord 1998;13:895-899.

10. Lees AJ, Blackburn NA, Campbell VL. The nighttime problems of Parkinson's disease. Clin Neuropharm 11:512, 1988.

11. Gorayeb I, Yekhlef F, Chrysostome V, Balestre E, Bioulac B, Tison F. Sleep disorders and their determinants in multiple system atrophy. J Neurol Neurosurg Psychiat 2002;72:798-800.

12. Diederich NJ, Comella CL. Sleep disturbances in Parkinson's disease. In: Chokroverty S, Hening WA, Walters AS. Sleep and movement disorders, 1st ed. Philadelphia: Butterworth Heinemann. 2003:478-488.

13. Laihinen A, Alihanka J, Rautasuo S, Rinne UK. Sleep movements and associated autonomic nervous activities in patients with Parkinson's disease. Acta Neurol Scand 1987;76:64-68.

14. Weller C, Bowes SG, Kirk CAA, Nicholson PW, Dobbs RJ, Dobbs SM. Measurement of axial rotation: Its relevance to screening for night-time hypokinesia in old age and parkinsonism. Age and Aging 1991;20:3-7.

15. van Hilten JJ, Weggeman M, van der Velde EA, Kerkhof GA, van Dijk JG, Roos RAC. Sleep, excessive daytime sleepiness and fatigue in Parkinson's disease. J Neural Transm [P-D sect] 1993; 5:235-244.

16. Gilman S, Low PA, Quinn N, Albanese A, Ben-Shlomo Y, Fowler CJ. Consensus statement on the diagnosis of multiple system atrophy. J Neurol Sci 1999;163(1):94-98.

17. Rechtschaffen A, Kales A, eds. A manual of standardized terminology, technique and scoring system for sleep stages of human subjects. Los Angeles, CA: Brain Information Service/Brain Research Institute, UCLA, 1968. 